\title{
Die Behandlung der acuten und subacuten Gonorrhoea anterior mit rückläufigen Einspritzungen stärkerer Silberlösungen.
}

\author{
Von \\ Dr. med. Hermann G. Klotz, \\ ordinirendem Arzt am Deutseben Hospital, New-York.
}

So lange bei den bestehenden socialen Zuständen nicht zu erwarten ist, dass Infectionen mit Gonorrhoe aufhören oder wesentlich eingeschränkt werden dürften, wird der Arzt zur Verhütung der traurigen Folgen dieser Krankheit immer noch am meisten beitragen können, wenn er die Wirkungen der Infection möglichst rasch and sicher beseitigt, und somit die Gefahr einerseits für die Ausdehnung der Infection auf die anliegenden; namentlich drüsigen Organe, andererseits für die Weiterverbreitung auf andere Personen vermindert. So lange ist wohl auch der Einzelne nicht nur berechtigt, sondern verpflichtet, wenn er durch eine Behandlungsmethode günstige Erfolge zu erzielen durch längere Prüfung derselben sich überzeugt hat, dieselbe zu allgemeinerer Kenntniss zu bringen und deren Anwendung zu empfehlen. In diesem Sinne möchte ich hier eine Behandlung beschreiben, die sich in meiner Praxis in Laufe der Jahre zu einer mehr weniger bestimmten Methode entwickelt und einer grossen Anzahl von Patienten, vornehmlich in der Privatpraxis, sowie mir selbst befriedigende, in mehreren Fällen selbst überraschende Resultate ergeben hat. Wie sich zeigen wird, macht dieselbe keineswegs auf absolute Originalität Anspruch, beruht auch nicht auf wesentlich neuen 
Principien, empfiehlt sich aber, wie ich glaube, besonders durch die Finfachheit ihrer Ausführung, welche keine neuen umständlichen Instrumente oder Apparate, noch weniger Abfassung eigner Lehrbücher nöthig macht.

Bei unsern heutigen bestimmten Kenntnissen von der Natur der Gonorrhoe ist beinahe selbstverständlich das Problem für jede Behandlung, das die Krankheit erzeugende Virus, d. i. den Gonococcus Neisser möglichst rasch und sicher zu beseitigen, und die durch denselben hervorgerufenen Entzündungserscheinungen zum Schwinden zu bringen. Bei dem Versuche, eine solche Behandlung durchzuführen, finden wir uns sehr bald in einem Dilemma: diejenigen Mittel, welche dem ersten Zweck zu genügen im Staude wären, vermehren in der Regel die locale Entzündung oder schädigen direct die Schleimhaut dermassen, dass sie rasch wieder ausgesetzt werden müissen, während diejenigen, welche gegen die locale Entzündung wirksam sind, die Vitalität der Coccen wenig oder gar nicht beeinträchtigen. An dieser Schwierigkeit sind verschiedene Methoden, namentlich die sogenannten abortiven gänzlich gescheitert, andere haben nicht $\mathrm{zu}$ den erwarteten günstigen Resultaten geführt, andere erwiesen sich wohl als brauchbar aber keineswegs allen Anforderungen genügend. Der Gedanke liegt nahe, durch eine Vereinigung beider Classen von Mitteln das gewünschte Ziel zu erreichen, und ist auch verschiedentlich verfolgt worden bei der Auswahl neuer Mittel, z. B. des Ichthyols, ohne ein solches zu finden, das nach beiden Richtungen hin genügend wirksam wäre. Ich habe versucht dem Ziele näher zu kommen durch Einspritzungen möglichst starker gonococcentödtender Lösungen, meist von Silbersalzen, welche in mehr weniger langen $\mathrm{Z}$ wischenräumen von dem Arzte selbst vorgenommen werden, während der Patient inzwischen milde antiseptische und adstringirende, entzündungswidrige Einspritzungen in gewöhnlicher Weise anwendet, gelegentlich auch die üblichen inneren Mittel zu Hilfe nimmt. In einer nicht geringen Anzahl von Fällen ist es mir auf diese Weise gelungen, das zu erzielen, was Finger als das Ideal einer medicamentösen Behandlung bezeichnet: „einen eben erst beginnenden Ausfluss aus der Urethra, dessen blennorrhagische 
Die Behandlung der acuten und subacuten Gonorrhoea etc. 401

Natur die Anwesenheit der Gonococcen bezeugt, sofort dadurch zu hemmen, dass man den Gonococcus, das Virus, tödtet, und damit die Ursache des sich entwickelnden Processes behebt." Selbstrerständlich kann eine solche Behandlung keineswegs in allen Fällen eine ganz gleichmässige sein; sie muss sich im Einzelfalle dem Zustand des erkrankten Organs möglichst anpassen. Ehe ich die Einzelheiten des Verfahrens unter den verschiedenen Umständen angebe, will ich jedoch darlegen, dass sich die Behandlungsmethode nach und nach aus den Bedürfnissen der Praxis und der Erfahrung von selbst entwickelt hat, nicht wie sich aus den obigen Bemerkungen vielleicht erwarten liesse, das Resultat theoretischer Speculation gewesen ist.

In den 80er Jahren wurde ich durch das Studium von Jamin's Etude sur l'uréthrite chronique blennorrhagique (Paris, 1883) mit den Instillationen, wie sie von Jamin's Lehrer Guyon in die Behandlung der Urethritis posterior eingeführt worden waren, näher vertraut und begann dieselben in Anwendung zu ziehen, wenn auch meist mit etwas schwächeren Lösungen von Argent. nitric. $\left(\mathbf{1} / \mathbf{2}-1-1 \frac{112}{2} \%\right.$ ) als J a m in, der $2 \%$ als durchschnittliche Stärke empfiehlt. Nun erwähnt Jamin, dass man die Instillationen auch auf umschriebene Herde in der pars anterior, besonders im Bulbus anwenden könne, doch bedürfe es grosser Vorsicht, weil die Lösung von dort aus rasch die Harnröhre nach dem Meatus zu durchfliesse, ehe sie durch Zersetzung und Niederschlag auf den erkrankten Stellen unschädlich gemacht worden sei. Trotzdem legte sich mir die Versuchung sehr nahe, Einspritzungen solcher Lösungen von Arg. nitr. in den Bulbus anzuwenden in den nicht gerade seltenen Fällen, in denen die Behandlung mit den üblichen Einspritzungen nach einigen Wochen den Ausfluss anscheinend ganz zum Schwinden gebracht hat, derselbe aber rasch wieder auftritt, sobald die Einspritzungen ausgesetzt werden, oder wo auch am Morgen der Ausfluss nicht mehr an der Harnröhrenmündung erscheint, sondern erst durch Ausdrücken von den tieferen Partien der pars pendula oder vom Bulbus her zum Vorschein gebracht wird, ohne dass Symptome einer Urethritis posterior vorhanden sind. Ich überzeugte mich nun bald, dass Einspritzungen auch grösserer. Mengen (2 bis 
3 Ccm.) von $1 / 2$ bis $1 \frac{1}{2} \%$ Höllensteinlösungen, vermittelst der Guy on'schen Spritze in den Bulbus eingebracht und nach Zurückhalten während einiger Minuten, durch den Meatus ausfliessen gelassen, nicht nur einen viel geringeren Reiz auf die Schleimhaut der ganzen pars anterior ausübten als zu erwarten gewesen wäre, sondern auch vorzügliche Wirkung äusserten. Diese Erfolge bestätigten eine Erfahrung, die sich mir schon früher bei Versuchen mit Irrigationen der Harnröhre mit Lösungen von Sublimat, Kali hypermangan. und Arg. nitr. aufgedrängt hatte, dass im Allgemeinen eine medicamentöse Flüssigkeit von der Schleimhaut der Harnröhre weit besser vertragen wird, objectivundsubjectir weit geringere Reizung hervorruft, wenn sie ron hinten nach vorn als in umgekehrter Richtung rom Meatus aus gegen den Bulbus hin die Harnröhre durchfliesst. Ich bin nicht im Stande, eine wissenschaftliche Erklärung für diese Erscheinung beizubringen; vielleicht hat die krampfhafte Zusammenziehung der Harnröhrenmuskulatur damit zu thun, welche in der Regel dem Findringen reizender Flüssigkeiten von der Harnröhrenöffnung her energischen Widerstand entgegen setzt. Ich würde mich jedenfalls sehr bedenken, eine $1 / \mathbf{2} \%$ oder selbst $1 / 4 \%$ Lösung von Arg. nitr. mit der gewöhnlichen Spritze einzuspritzen, während dieselbe vom Bulbus aus nach rorn sich entleerend allerdings auch ein mehr weniger heftiges Brennen und eine vermehrte Secretion hervorruft, welche jedoch spontan innerhalb $1 / 4$ bis einer Stunde wieder verschwinden. Ich behandelte also die oben beschriebenen subacuten Fälle längere Zeit hindurch in dieser Weise mit recht gutem Erfolge und sah nur sehr selten Complicationen seitens der tiefen Harnröhre, der Prostata oder des Nebenhodens auftreten. Die Spritze, deren ich mich zumeist bediente, ist in Wirklichkeit eine etwas vergrösserte Ausgabe der Braun'schen Uterusspritze, welche in der voroperativen Periode der Gynäkologie vielfach benutzt wurde. Meine Spritze ist aus Hartgummi angefertigt mit ca. 3 Cubikcentimeter haltendem Glasrohr; dem in die Form eines abgestumpften Kegels ausgehenden Ende der Spritze wird ein ca. $18 \mathrm{Cm}$. langes, gegen das Ende zu etwas gebogenes Hart- 
Die Behandlung der acuten und subzaten G snorrioea etc. 403

gummirohr aufgesetzt, welches in einen aufgeschraubten, olivenförmigen:Knopf ron $\mathrm{Nr} .16$ bis $18 \mathrm{der}$ französischen Scala ausgeht. Die Oeffnung ist an der Seite des Knopfes angebracht, so dass das Einfliessen der Lösung in die urethr. posterior erschwert wird. Bei engem Meatus gebrauche ich die Ultzmann'sche oder Keyes'sche Metallspritze, deren Rohr ein Caliber ron ca. 14. Fr. hat (die Ke yes'sche Spritze unterscheidet sich ron der Ultzmann'schen durch zwei seitliche Metallflügel am Ende der Spritze selbst als Stützpunkte für die Finger). Jedoch habe ich bemerkt, dass die Beriihrung der Schleimhaut auch mit der erwärmten Metallspritze weit unangenehmer empfunden wird als die mit dem Gummirohr. In Ausnahmefällen musste ich mich feiner elastischer Katheter als Ansatzrohr bedienen. Die Spritze wird, nachdem das Rohr mit einem beliebigen Lubricans eingeschmiert ist (ausser wenn die einzuspritzende Flüssigkeit wie bei Argonin, Protargol und Albargin dies überflüssig macht, vorsichtig bis zum Bulbus eingeführt und dann langsam entleert, während die Finger der linken Hand die Harnröhrenmündung durch Zusammendrücken geschlossen halten. Hierauf wird die Spritze entfernt, die Harnröhre einige Zeit, nicht weniger als 2 Minuten, geschlossen gehalten, darauf die Lösung nach und nach herausfliessen gelassen und an der Oeffnung mit Watte aufgefangen. In der Regel werden auf diese Weise zwei Einspritzungen hintereinander vorgenommen; die glans penis wird zur Aufnahme der noch aussickernden Flïssigkeit mit absorbirender Baumwolle umwickelt. Brennen tritt meist erst nach dem Abfliessen der Lösungen auf, namentlich bei Argent. nitr., nur selten ist dasselbe so heftig gewosen, dass ich mich veranlasst gesehen habe, einige Tropfen einer höchstens $4 \%$ igen Cocainlösung einzuspritzen.

Trotz der guten Erfolge und der Abwesenheit unangenehmer Folgen hatte ich mich doch nicht entschliessen können, das Verfahren auch auf friihere Stadien der Gonorrhoe auszudehnen, wo noch lebhaftere Entzündungserscheinungen vorhanden waren. Da erschien 1894 (Monatshefte für praktische Dermatologie XVIII, pag. 596) von Sehlen's Artikel: „Zur Frühbehandlung der Gonorrboe ${ }^{a}$, der bereits auf dem 4 . Congress 
der Deutschen Dermatologischen Gesellschaft zum Vortrag angemeldet gewesen war, und im Auszug in den Verhandlungen des Congresses abgedruckt ist.

Von Sehlen behauptet, dass "die Heilungsdaner der Gonorrhoe bei entsprechender Behandlung in directer Abhängigkeit von der Infectionsdauer steht, $\mathbf{d}$. h. der Zeit, welche vom Momente der Infection bis zum Beginn der Behandlung verflossen ist". Daher müsse die Therapie möglichst früh eingreifen. Vermittelst einer sehr übersichtlichen tabellarischen Zusammenstellung von 20 Fällen suchte er recht augenfällig darzustellen, dass der Tripper unter günstigen Umständen in einem Tage heilbar sei, wenn die Behandlung bis spätestens 4 Tage nach der Ansteckung einsetze, dass auch noch 8 Tage nach der Ansteckung in geeigneten Fällen Heilung in 3 bis 6 Tagen erfolgen könne, dass aber 8 bis 14 Tage nach der Ansteckung die Krankheit eine länger fortgesetzte Behandlung erfordere. "Die Frühbehandlung sei im Stande, die Verbreitung des Tripperprocesses auf weitere Strecken der Harnröhre zu verhindern und den Verlauf zu einem milden, auf die vorderen Abschnitte der Urethra beschränkten zu gestalten, selbst wenn das vollständige Verschwinden der Gonococcen nicht alsbald erreicht werde." Die von ihm angewandte „Frühbehandlung soll erstreben, dass der Erreger der Krankheit möglichst rasch und sicher getödtet werde, ohne dass das erkrankte Organ darunter leidet". Zur Ausführung dieses Zweckes bedient sich von Sehlen des altbewährten Arg. nitr., aber nicht in $2 \%$ Lösungen, wie es von verschiedenen Franzosen und auch von Welander angewandt wurde, sondern da das Arg. nitr. noch in Verdünnungen bis $1: 10.000$ antibakteriell wirksam sich erwiesen, suchte er für jeden Fall, entsprechend dem Entzündungsgrade der Schleimhaut die passende Concentration auszuwählen, von der eine genügende antigonorrhoische Wirksamkeit auch in tiefere Gewebsschichten hinein garantirt ist und eine allzuheftige Einwirkung auf die entzündete Schleimhaut vermieden wird. Dementsprechend wählte er Lösungen von 1:1000 an and spritzte dieselben in frischen Fällen mit einer kleinen Injectionsspritze ein, so dass die vordere Harnröhre prall damit gefüllt wurde, vertheilte den Inhalt durch reibende Bewegungen, so dass jeder Punkt der Schleimhant auf das innigste mit dem Medicament in Berührung gebracht wird und liess dann die Flüssigkeit wieder ablaufen. Diese Manipulation wird mehrere Male wiederholt bis die sichtbare Oberfäche der Schleimhaut durch Silberniederschläge weisslich verfärbt erscheint. Die Fossa wird ohne Anwendung von Gewalt und ohne Instrumente noch mit einer stärkern Lösung bis zu $2 \%$ eingerieben und dann soweit zugänglich mit Europhenpulver betupft. In vorgeschrittenen Fällen beginnt er mit Spülungen der vordern Harnröhre durch den Rücklauf catheter mittelst Lösungen von $1: 10.000$ bis $1: 1000$. Die Harnröhre gewöhnt sich meist auffallend rasch an stärkere Concentrationen, und nach wenigen Tagen kann man schnell zur Applicationen von $1 / 4$ bis $1 \%$ Lösungen durch die Spritze steigen. 
Ich bin etwas ausführlicher auf von Sehlen's Arbeit eingegangen, weil dieselbe auffällig wenig Beachtung gefunden zu haben scheint, unter andern auch von $\mathrm{F}$ ing er in der neuesten Auflage seines Lehrbuchs (1900) gar nicht erwähnt wird. Der Tod des Verfassers bald nach der Veroffentlichung des Artikels mag wohl etwas damit zu thun gehabt haben, vielleicht auch der Umstand, dass gerade um diese Zeit die von J a n e t neuerdings wieder eingeführte Irrigationsmethode von verschiedenen Seiten in ungewöhnlich energischer Weise in den Vordergrund gedrängt, von Vielen mit grosser Begeisterung aufgenommen wurde. Daher möchte ich die Aufmerksamkeit der Collegen wiederum auf von Sehlen's Arbeit lenken, umso mehr als ich seine Behauptungen fast vollständig bestätigen kann, und meinerseits dem Gefühl der Dankbarkeit und Verpflichtung Ausdruck geben für die Anregung und Ermunterung, die ich durch dieselbe erhielt.

In der That fing ich alsbald an, seinem Beispiele zu folgen, und wenn ich seine Methode auch in verschiedener Weise modificirt habe, so bin ich doch in der Hauptsache seinen Principien gefolgt. Gestütat auf meine bereits mitgetheilten Erfahrungen habe ich von vornherein davon abgesehen, mich der gewöhnlichen Injectionsspritze zu bedienen, sondern habe die oben beschriebene Spritze benutzt und die Lösungen vermittelst derselben möglichst hinter die inficirten Partien der Harnröhre gebracht, d. $h$. in sehr frühen Fällen bis in die Mitte der pars pendula, in späteren bis zum Bulbus. Ich glaube nicht, dass damit einer Verschleppung der Gonococcen nach hinten Vorschub geleistet wird, namentlich wenn man, sobald die Fossa passirt ist und die Harnröhrenmündung mit den Fingern verschlossen worden ist, anfängt tropfenweise die Lösung ausfliessen zu lassen, so dass die Spitze des Ansatzrohrs von der Lösung umspült vorwärts bewegt wird. Nach der Entfernung der Spritze habe ich ein ron Sehlen's ähnliches Verfahren eingeschlagen, von dem Auswischen der Fossa jedoch abgesehen. Nur äusserst selten ist es mir wegen des Schwellungsgrades der Schleimhaut nicht möglich gewesen, die Einspritzung auszuführen.

Eine wesentliche Förderung wurde dieser Behandlungsmethode zu Theil durch die Finführung der verschiedenen neuen Silberverbindungen in die Praxis, indem bei der Herstellung derselben darauf hingestrebt wurde, die reizenden Eigenschaften herabzusetzen, das Coaguliren durch Chloride, Albuminate etc. zu vermeiden und somit die Tiefenwirkung auf die; 
Gewebe zu erhöhen. Bei der grossen Anzahl dieser Mittel, die es dem Einzelnen kaum gestattet, ein jedes derselben genügend zu prüfen, hat sich meine persönliche Erfahrung hauptsächlich auf Argonin, Protargol und seit dem Frühjahr 1901 besonders auf Albargin beschränkt, doch zweifle ich nicht, dass die andern wie Argentamin, Largin, Ichthargan; Itrol etc. sich ebenso leicht dem Verfahren anpassen würden. Die geringere Reizwirk ung und Schmerzhaftigkeit dieser Mittel gegenüber dem Argent. nitric., die wohl kaum angezweifelt werden kann, machten dieselben besonders geeignet für die Anwendung rerhältuissmässig starker Lösungen. Argonin bat sich mir als das mild este unter denselben erwiesen; in $10 \%$ Lösung verursachte es kaum mehr als ein leichtes Brennen auch bei ziemlich entzündeter Schleimhaut; Protargol wurde in 2 bis $4 \%$ Lösungen gut vertragen, obwohl es meist ein etwas intensiveres, immerhin mässiges und kurzdauerndes Brennen verursacht. Einer eingehenden Prüfung habe ich seit dem letzten Frübjabr das Albargin, Silbergelatose, unterworfen und zwar mit sehr befriedigendem Erfolge. Es wurde $1 \%$ und $2 \%$, während der letzten Wochen auch $5 \%$ Lösung benutzt, meistens in der Stärke von $2 \%$ auch noch bei ziemlich deutlichen Entzündungserscheinungen. Es bewirkt wie Protargol anfangs Brennen, das aber rascher als bei jenem vorüber geht. Vor der Einführung der neueren Silberrerbindungen habe ich mich bei heftigerer Entzündung mit gutem Erfolge manchmal des Thallin. sulphur. bedient und zwar in der gonococcentödtenden Stärke von $7 \%$. Dasselbe verursacht momentan allerdings recht lebhaftes Brennen, das aber bald einer schmerzstillenden, beinahe narkotisirenden Wirkung Platz macht; es empfiehlt sich dasselbe daher besonders bei grosser Empfindlichkeit der Harnröhre. Allein die schlechte Haltbarkeit der Lösung, die nach und nach eine ganz tief rothbraune Farbe und stark adstringirende Eigenschaften annimmt, der ziemlich hohe Preis des Mittels und der Umstand, dass es die Wäsche dauernd beschmutzt, machen das Thallin weniger angenehm im Gebrauch. Ichthyol habe ich his zu 5\% Verdünnung angewandt aber ohne besonderen Erfolg.

Es ist bei der Beurtheilung der verschiedenen Silberverbindungen von manchen Seiten besonderes Gewicht auf den 
Silbergehalt derselben gelegt worden. Demnach würde das $9 \%$ Silber (gleich dem Argentamin als Argent. nitric.) enthaltende Albargin dem Protargol überlegen sein. Indessen glaube ich nicht, dass der Silbergehalt allein bestimmend ist, sondern vielmehr, dass neben den chemischen insbesondere auch die physikalischen Eigenschaften eine Rolle spielen dürften. Es ist geltend gemacht worden, dass die Lösungen der Albuminate wie die der Gelatose durch Chloride, Albuminate und verdünnte Säuren nicht gefällt werden. Auf Grund dieser Eigenschaft hat man für dieselben eine unbegrenzt tief in die Gewebe eindringende Wirkung angenommen; ob eine solche Tiefenwirkung durch genaue Experimente wirklich nachgewiesen worden ist, darüber habe ich nirgends bestimmte Angaben finden können. Dagegen scheinen die nach Bornemann von Liebrecht gemachten Versuche über die Dialysirfähigkeit von Protargol und Albargin allerdings entschieden zu Gunsten des letzteren ausgefallen zu sein. Vom rein praktischen Standpunkt, aus möchte ich wohl glauben, dass die dünnen, leicht beweglichen Lösungen des Albargin eher geeignet sein dürften in die Gewebe einzudringen, als die mehr weniger klebrigen Lösungen von Protargol und besonders von Argonin. Dagegen spricht meine Erfahrung auch wieder dafür, dass schliesslich die Lösungen des Argent. nitric. trotz der Niederschläge sich unter verschiedenen Verbältnissen doch am wirksamsten erweisen, umsomehr je länger die Erkrankung bestanden hat.

Wenn im Vorhergehenden die geringere Reizwirkung der neveren Silberpräparate hervorgehoben worden war, so muss doch ein gewisser Entzündung erregender, Secretion vermehrender Einfluss auch für die mildesten dieser Präparate zugegeben werden. Wie die Beschaffenheit der dem Auge unmittelbar zugänglichen Scbleimhaut der Lippen der Harnröhrenöffnung unschwer erkennen lässt, äussert sich dieselbe durch Schwellung und vermebrte Röthe derselben, wenn auch häufig nur kurz andauernd. Dass vermebrte Blutzufuhr und vermehrte Durchtränkung der Gewebe für die Entwicklung von Bakterien im Allgemeinen einen günstigeren Boden darstellen als eine gesunde Schleimhaut mit unbeschädigtem Epithel und geringer Gefässfüllung ist wohl kaum in Abrede zu stellen. Daher erscheint 
es trotz des Nachweises, dass die Gonococcen sehr rasch die Gewebsschichten durchdringen können, in hohem Grade wünschenswerth, dass nicht nur die natürliche, die Cocceninfection begleitende, sondern auch die künstlich durch die antibakteriellen Massnahmen hervorgerufene locale Entzündung möglichst eingeschränkt, beziehentlich beseitigt werde. Dazu dienen im Allgemeinen adstringirende und antiseptische Lösungen, und es lag nahe, die Silbereinspritzungen nur in längeren Pausen anzuwenden, um eine cumulative Reizwirkung zu vermeiden, diese Pausen aber durch derartige adstringirende, in der gewöhnlichen Weise vorgenommene Einspritzungen auszufüllen. Diese müssen natürlich auch in jedem einzelnen Falle dem jeweiligen Zustand der Schleimhaut angepasst werden. Im Allgemeinen gebe ich für diesen Zweck solchen Einspritzungen den Vorzug, welche Niederschläge bilden. Dieselben liefern einerseits ähnlich den Streupulvern auf der äusseren Haut eine schützende, die gegenüberliegenden Flächen von einander trennende Decke, andererseits dürften sie wohl auch den auf der Oberfläche befindlichen Gonococcen wenigstens die Weiterentwicklung erschweren. Am häufigsten bediene ich mich folgender Vorschrift: Rp. Acid. borici $1 \cdot 5$

Plumbi acetic.

Zinc. sulphuric $\bar{a} \bar{a} \quad 0.75$

Glycerin $5 \cdot 0$

Aquae $120 \cdot 0$

die ich fernerhin kurz als Injection B.P.Z. bezeichnen werde.

Finger in seinem Lehrbuche verwirft absolut die locale Behandlung mit einfachen oder antiseptischen Adstringentien im frühen Stadium. "Dieselbe," sagt er (pag. 151), "ist hier nicht am Platze, da wir in diesem Stadium das Hauptaugenmerk auf die Elimination der Gonococcen richten müssen, dagegen die entzündlichen Erscheinungen, die zur Elimination der Gonococcen beitragen, insbesondere die Secretion nicht zu tief herabgesetzt werden soll, weil dadurch gerade das Zurückbleiben der Gonococcen in der Tiefe ermöglicht und gefördert werde." Abgesehen davon, dass sich in der von Finger für die frühen Stadien der Gonorrhoe empfohlenen Bebandlung ausser vermehrter Diurese überhanpt nirgend etwas findet, was die Elimination der Gonococcen befördern könnte, trotzdem dass er das Hauptaugenmerk auf diesen Punkt gerichtet haben will, kommt es allerdings viel darauf an, was man unter "frühem Stadium " versteht. Zugegeben, dass die Gonococcen sich nicht auf der Ober- 
fläche und in den obersten Schichten der Epithelien vermehren, sondern erst zwischen dieselben eindringen müssen, ehe sie stärkere Entzündungserscheinungen hervorbringen, so besteht sicherlich in den ersten Tagen nach der Infection, wann die Secretion überhaupt gewöhnlich noch eine geringe ist, eine solche Gefahr nicht; ursprünglich und für eine gewisse Zeitperiode müssen sich die Gonococcen auf der Oberfl̈che oder in deren Nähe aufhalten, und können somit noch nicht in der Tiefe zurückgehalten werden. Gelingt es nun in dieser Periode za verhindern, dass Entzündungserscheinungen: vermehrter Blutzufluss und Durchtränkung der Gewebe, überhaupt auftreten oder irgend welche Intensität erreichen, so wird sicherlich dadurch demEindringen derGonococeen in dieTiefe einHinderniss in den Weg gelegt. Dies muss aber noch in höherem Grade der Fall sein, wenn gleichzeitig oder vorher eine nicht nur gonococcentödtende sondern auch eine die von $F$ inger als so wünschenswerth bezeichnete Secretion wenigstens temporär befördernde Silberlösung applicirt wird. Sagt er doch selbst (p. 144) von der medicamentösen, vorwiegend topischen Behandlung, dass "sie die Aufgabe hat, den Kampf zwischen Gonococcen und Schleimhaut dadurch abzukürzen, dass sie die Gonococcen angreift, die Schleimhaut unterstützt und widerstandsfähiger macht." Dass aber eine entzündete Schleimhaut einen weit besseren Boden für die Entwicklung und Vermehrung der Gonococcen darbietet, erhellt ohne Weiteres aus dem wohl allgemein anerkannten und verbreiteten Vorgehen in Fällen von latenter oder anscheinend geheilter Gonorrhoe, absichtlich durch Injection von Argent. nitric. eine acute Entzündung temporär hervorzurufen, unter deren Einfluss in den tiefern Schichten sich aufhaltende Gonococcen auf die Oberfäche gelangen und zur Vermehrung Gelegenheit bekommen. Diese Erfahrung steht auch in Widerspruch mit der von Fing e r (p. 151) ausgesprochenen Ansicht, dass im 2. Stadium der Krankheit, wann er selbst die antiseptischen Adstringentien angewandt haben will, die Hauptmasse der Gonococcen oberflächlich liege. Wenn das wirklich der Fall wäre, so würden wohl die chronischen, immer und immer wieder recidivirenden Fälle weit weniger zahlreich sein, und dass diese nach der zuwartenden Behandlung nicht aufträten oder weniger häufig wären, wird wohl Niemand behaupten wollen. Aber auch wenn man zugeben will, dass der frühe Gebrauch der Adstringentien einen gewissen nachtheiligen Einfluss gegen die Eliminirung der Gonococcen ausüben könne, so würde ich doch die Einschränkung der Absonderung, wenn dieselbe durch Adstringentien ohne andere Nachtheile erzielt werden kann für höchst wünschenswerth erachten schon aus Rücksicht auf den Comfort des Patienten, der doch anch eine gewisse Beachtung beanspruchen darf. Dieselbe befördert nicht nur die Reinlichkeit sondern verringert auch die Gefahr der Weiterverbreitung der Infection, ist somit von Wichtigkeit für die private wie für die öffentliche Hygiene. Auch stehen die subjectiven Symptome des Patienten im Allgemeinen wohl vielmehr in geradem Verhältnisse zu dem Grade der Entzündungserscheinungen als $z u$ der Menge der anwesenden Gonococcen. Trotzdem 
bin ich keineswegs der Ansicht, dass adstringirende Einspritzungen unter allen Umständen vorzunehmen seien.

Nach diesen Auseinandersetzungen erübrigt es nun, die praktische Anwendung der Methode unter verschiedenen Umständen anzugeben und durch Beispiele zu erläutern. Es ist nicht meine Absicht, alle von mir so behandelten Fälle oder auch nur eine grössere Anzahl derselben zusammenzustellen, zumal es mir an der Zeit fehlen würde, alle Beobachtungen aus meinen Krankenjournalen berauszusuchen. Ich glaube nicht, dass solche massenhafte Krankengeschichten eine grössere Beweiskraft ausüben würden, selbst wenn der Leser sie alle genau durchlesen wollte, ebensowenig, dass Versuche, statistische Zusammenstellungen ïber die Zeit des Verschwindens der Gonococcen zu geben, von besonderem Werthe sein würden. Häufig verschwindet die Secretion in wenigen Tagen vollständig, so dass der Nachweis der Anwesenbeit oder Abwesenheit der Coccen unmöglich wird. Trotzdem wird der Sicherheit wegen die Behandlung fortgesetzt und mag so die Gegenwart der Coccen wesentlich überdauern. Ich behaupte nur, dass eine nicht geringe Anzahl von Fällen, welche früh in Behandlung kamen, in wenigen Tagen dauernd geheilt wurden, dass in anderen die Beseitigung der Coccen zwar nicht sogleich erreicht wurde, die Erscheinungen der Krankheit aber aut einem so geringen Grad beschränkt blieben, dass die Patienten so gut wie gar keine Unannehmlichkeiten oder Beschwerden hatten als die mit der Behandlung selbst im Zusammenhang stehenden, und dass nur in einer geringen Anzahl das Weiterschreiten der Infection auf die hintere Harnoöhre ebenso wie Complicationen seitens des Nebenhodens, der Blase und der Prostata nicht verhindert werden konnten. Sehr copiöse Ausflüsse, bedeutendere Schmerzen bei der Urinentleerung, Störung der Nachtruhe durch schmerzhafte Erectionen, Lymphangitis und ähnliche besonders lästige Symptome kommen nur höchst selten zur Beobachtung, oder werden, wenn sie im Anfange der Behandlung mehr weniger rorhanden waren, gewöhnlich innerhalb einiger Tage auf ein Minimum reducirt. Dass die Behandlung nicht in allen Fällen anwendbar ist und nicht immer zu dem gewünschten raschen Resultate fübrt, gebe ich gern $z u$, auch abgesehen von 
solchen Patienten, welche durch Indiscretionen wie Nachlässigkeit in der Behandlung, Trinken, geschlechtliche Aufregung oder selbst Coitus vor erreichter Heilung, übermässige körperliche Anstrengungen etc. die Bemühungen des Arztes vereiteln.

Es ist kaum nöthig zu erwähnen, dass ich nur solche Fälle berücksichtige, in denen der Charakter der Krankheit als Gonorrhoe durch den mikroskopischen Nachweis der Gonococcen vermittelst der üblichen Färbemethoden festgestellt worden ist. Nicht so einfach ist die Entscheidung der Frage, wann ein Fall von Gonorrboe als geheilt zu betrachten sei, umso mehr als es in der Praxis in der Regel nicht möglich ist, alle Patienten so lange unter Beobachtung zu behalten als es wohl mit Heiratscandidaten vielleicht durchfübrbar ist, oder gar sie bis an ihr Lebensende zu verfolgen, wie es nach den Ansichten Einiger beinahe nothwendig erscheinen würde. Man muss sich da doch auf einen praktischen Standpunkt stellen und den Kranken als geheilt betrachten, wenn Absonderung und Gonococcen völlig verschwunden sind und verschwunden bleiben, nachdem jede Behandlung ausgesetzt und der Kranke mehrere Wochen seine gewohnte Lebensweise, namentlich betreffend den Genuss geistiger Getränke wieder aufgenommen hat. In der Privatpraxis namentlich kann man mit grösserer Bestimmtheit eine Heilung als sicher annehmen, wenn der Patient sich nicht weiter blicken lässt. Betreffend die sogenannten Urethralfäden so bin ich gleich manchem Anderen durch langjährige Erfahrung zu der Ueberzeugung gekommen, dass es nicht in allen Fällen möglich ist dieselben vollständig zu beseitigen, und dass die Versuche, dies zu erreichen oft mehr Schaden als Nutzen bringen, ferner dass das Fortbestehen derselben weder für die Männer selbst noch für die Frauen mit welchen sie geschlechtlichen Umgang haben, die geringsten Nachtheile bringt, selbst wenn dieselben neben Epithelien noch Eiterkörperchen in geringer Anzahl enthalten. Daher kann ich auch der Ansicht Leven's nicht beipflichten, dass, so lange Eiterkörperchen sich vorfinden, man die Anwesenheit ron Gonococcen anzunehmen habe. Die verschiedenartigsten Schädlichkeiten sind im Stande, auf der Schleimhaut der Harnröhre die Production von Eiterkörperchen hervorzurufen und zu unterhalten, mögen nun zu einer Zeit 
oder niemals Gonococcen auf derselben vorhanden gewesen sein. Ich bin überzeugt, dass Leven bald genug selbst diesen Standpunkt wieder verlassen wird.

Dagegen möchte ich aufmerksam machen auf das verschiedene Verhalten der primären, ersten Infectionen eines Individuums durch den Gonococcus gegenüber den Reinfectionen solcher, die bereits vor längerer oder kürzerer Zeit eine Gonorrhoeinfection überstanden haben, auch wenn dieselben sicher geheilt waren. Im Allgemeinen sind die Ersteren, vielleicht weil sie gewöhnlich auch eine längere Incubationsdauer aufweisen, bedeutend mehr geneigt, heftige acute Entzündungserscheinungen hervorzurufen und der Behandlung weit grössere Schwierigkeiten und hartnäckigeren Widerstand entgegenzustellen. Dieser Umstand ist besonders zu berücksichtigen bei der Beurtheilung der Wirksamkeit gewisser Behandlungsmethoden und namentlich einzelner Arzneimittel. Auch wenn man aus einem grösseren Beobachtungsmaterial statistische Zusammenstellungen macht und daraus Schlüsse ziehen will, sollten die primären Infectionen von den secundären getrennt beurtheilt werden. Die Ersteren sollten den besten Prüfstein für die Beurtheilung abgeben. Leider habe ich selbst nicht häufig Gelegenheit, Patienten in den ersten Stadien ihrer ersten Gonorrhoeinfection zur Behandlung zu bekommen. Haben doch die meisten solcher Patienten keine Ahnung von der Bedentung der Krankheit und versuchen daher sich von guten Freunden, Apothekern oder Quacksalbern Hilfe zu verschaffen, im günstigen Falle wenden sie sich an den Hausarzt, an die Specialärzte aber erst, wenn sie nach längerer Zeit nicht die erwünschten Erfolge sehen. Doch verfüge ich über einige Beobachtungen, die wenigstens theilweise kurzer Berichterstattung werth erscheineu, und am besten mein Verfahren därstellen.

Fall 1. O. R., 21 Jahre alt, consultirte mich am 20. August 1899 wegen geringer Entzündungserscheinungen an der Harnröhrenmündung, ohne je vorher geschlechtlich krank gewesen zu sein. Die geringe schleimige Absonderung erwies sich frei von Eiterzellen und Gonococcen oder anderen Bakterien und schien durch die mechanische Reizung durch ein. sehr straffes Frenulum hervorgerufen zu sein. Sie war ohne weitere Behandlung nach einigen Tagen verschwunden.

1900. 13. Juli Morgens. Nach Coitus am 9. gestern Absonderung bemerkt, jetzt typische Gc. in- und ausserhalb von Eiterzellen, Injection von 
Die Behandlung der acuten und subacuten Gonorrhoea etc. 413

Protargol $2 \%$ und $4 \%$ je eine Spritze voll bis in die Mitte des pars pendula, mässiges Brennen. Inj. B. P. Z. gegen Abend und vor Schlafengehen selbst zu machen.

14. Juli. M. Spur von Feuchtigkeit, Urin völlig klar, Protarg. $2 \%$ u. $4 \%$ kaum empfindlich, Inj. B. P. Z. wie gestern, und 3 Mal am 15. zu machen.

16. Juli desgleichen.

18.,NII. 3 Stunden nach Entleerung des Harns kein Secret, Urin ganz klar mit einer längern, dünnen Faser. Inj. Arg. nitr. $1 / 4 \%$ und $1 / 2 \%$ Fortsetzen der Einspritzung mit B. P. Z. bis zum 20. Abend.

21./VII. Spur von Feuchtigkeit, Urin klar mit einigen ganz feinen Fasern. Inj. Arg. nitr. $1 / 1 \%$ and $1 / 2 \%$; Einspritzung bis zum 22 . Abend.

24./VII. Eine Spur von Schleim frei von Eiterzellen und Cocoen jeder Art. Inj. Arg. $1 / \%$ and $1 / \%$. Am Abend noch eine Einspritzung, dann jede Behandlung sistirt.

Im September 1900 hatte ich Gelegenheit, die dauernde Heilung zu constatiren bei Gelegenheit einer Consultation wegen eines Furunkels. Der Termin des Verschwindens der Gonococcen ist nicht festzustellen, wohl aber definitive Heilung innerbalb 11 Tagen, während welcher Patient kaum irgend welche Besehwerden empfunden hatte.

Fall 2. B. K., 28 Jahre, früher nie geschlechtlich krank, Bruder eines Patienten, den ich längere Zeit an chron. Gonorrh., später an verschiedenen frischen Infectionen behandelt hatte. B. K. bemerkte am 9. April 1901 nach Coitus am 6. geringe Absonderung bei mässiger Empfindlichkeit der Harnröhre beim Uriniren. Geringe Menge eitrigen Secrets mit reichlichen typischen G. C. in u. ausser Zellen, Urin klar. Inj. Protarg. $2 \%$ n. $4 \%$, ziemlich starkes Brennen von kurzer Dauer. Einspritzung von B. P. $Z$.

10./IV. Gestern noch ziemlich empfindlich beim Uriniren und beim Einspritzen gewesen, heute Morgen schmerzfrei, 11/2 Stunde nach Uriniren Spur yon Secret, neben Eiterzellen ziemlich reichliche Plattenepithelien und einige Gruppen von G. C. zwischen den Zellen, eine G. C. einschliessende Zelle. Urin klar. Protarg. 2 und $4 \%$, Einspritzung B. P. Z.

11./IV. Am Morgen vor Entleerung des Urins nur etwas weissliches Secret bestehend aus dem Niedersehlag der am Abend gemachten Einspritzung. Urin klar, 2 Spritzen Protargol 4\%. Einspritzung B. P. Z. bis zum 12. Morgens, keine subjectiven Beschwerden.

13./IV. Ziemlicher Tropfen sehr dünnen Secrets, 2 Spritzen Povtargol $4 \%$.

16./IV. Gar keine subjectiven Beschwerden, nach 5 Stunden seit Harnentleerang eine kleine Menge Secret, bestehend grösstentheils aus Eiterzellen, Epithelien, zwischen denselben kleinere und grössere Gruppen von Diplococcen, einige grössere Conglomerate anderer Coccen. Inj. Arg. nitr. $1 / 2 \%$, Einspritzen bis zum 18. Abends. 
19./VV. bis gestern nichts zu sehen gewesen, heute Morgen Spur von Secret mit einzelnen Gc.-haltigen Zellen, wenige freie Coccen, Urin in beiden Portionen klar; 2 Spritzen Arg. nitr. 1\%.

20./IV, nur Spur von Secret, ohne irgendwelche Coccen, erster Urin ein wenig trüb. Inj. Arg. nitr. $1 \%$, Einspritzung bis zum 21. Abends.

24./IV. bis gestern nichts zu sehen gewesen, heute Morgen etwas Secret mit typischen, auffällig kleinen und dünnen G. C. innerbalb und ausserhalb der spärlichen Eiterzellen, einzelne Epithelien. Inj. Albargin $2 \% 2$ Spritzen. Einspritzen bis zum 25. Abend.

27./IV. nach reichlichem Biertrinken gestern Abend eitriger Tropfen mit zahlreichen typischen G. C. in und ausser Zellen, zweite Urinportion völlig klar. Albargin 2\%2 Spritzen, Einspritzung bis zum 29. Abend.

30./IV. nach Aussetzen der Einspritzung ein wenig Secret mit spärlichen G. C. in und ausser Zellen, erster Urin noch ein wenig trüb; 2 Spritzen Arg. intr. $1 / 2 \%$. Einspritzung von Kali hypermanganic. 0.05 : $125 \cdot 0$.

3./ V. gelegentlich etwas Brennen in der Harnröhre, geringe wässrige Absonderung, noch spärliche Eiterzellen aber keine G. C., erste Urinportion ganz geringe Trübung; 2 Spritzen Arg. nitr. 1/2\%. Einspritzung B. P. Z. bis zum 5. Abend, dann Behandlung aufhören.

11./V. nach Coitus am 7. etwas reichlicher Ausfluss, nur wenige Stunden anhaltend, seitdem nur etwas feucht geblieben, sehr geringe Menge graugelben Secrets, $z$ wischen den wenigen Zellen eine geringe Anzahl Diplococcen nicht ganz typischer Form. 2 Spritzen Albargin 2\%, noch einige Tage Einspritzung von Kali hypermang. Nach Aussetzen der Behandlung kein Wiederauftreten von Secret oder andern Symptomen.

Der Fall zeigt die Hartnäckigkeit der Gonococcen bei einer nicht allzukurzen, immerhin wenig über 4 Wochen betragenden Behandlungsdaver trotz versehiedener Indiscretionen. Dagegen ist zu beachten die Abwesenheit fast jeden Symptoms, die allerdings auch als Schattenseite die Neigung des Patienten zu frühzeitigen Excessen zur Folge hat.

F a 11 3. A. B., 27 Jahre alt, früher nie an Gonorrhoe erkrankt geweses, bemerkt vor ca. 6 Tagen, 9 Tage nach letztem Coitus, etwas Absonderung, hat seit einigen Tagen Kal. hypermang, eingespritzt.

25. September 1901 ziemlich reichliches, weissliches Secret mit typischen G. C. haltenden Zellen in mässiger Anzahl. Inj. Albargin 1\% und $2 \%$. Einspr. B. P. $Z$.

26./IX. bedeutende Besserung, orificium urethrae noch etwas roth und infiltrirt, wenig transparentes Secret mit einzelnen G. C. haltigen Zellen, Urin klar. $2 \%$ Albargin 2 Spritzen.

27./IX. orific: weich, noch etwas roth, Spur von klarem Schleim: Eiterzellen einige wenige Exemplare von G. C. enthaltend, einige Gruppen ausserhalb, ziemlich reichlich Epithelien, Urin klar, ein Faden. 2 Spritzen $2 \%$ Albargin. 
28./IX, Aussehen gut, aber etwas Secret mit G. C. haltigen Zellen im hintern Theil der Harnröhre, erste Urinportion trüb, Albargin $2 \% 2$ Spritzen in die Urethra posterior.

30./IX. keinerlei subjective Beschwerden, etwas Secret mit reichliche G. C. enthaltenden Zellen, etwas blutig, Urin ein wenig trüb, Arg: $1 / 4 \%$ membran, $1 / 2 \%$ in Bulbus.

1./X. bis 3./X. Secret in geringer Menge fortwährend etwas blutig, ohne subjective Beschwerden; G. C. in kleiner Zahl enthaltend, Urin trüb. Inj. Árg. nitr. $1 / 4 \%$ und $1 / 2 \%$ in membr. resp. Bulbus, vom 2. ab Ol. Santali.

4./X. bis 9./X. Trübung des Urins abnehmend, ebenso Secret und Gonococcen allmählig reducirt unter Irrigation der Blase und Harnröhre vermittelst Nélaton Catheter mit Arg. intr. 1: 1000 und 1:750 am 4., 5., 7. und 9. October, am letzten Tage eine Spur von Secret, Urin ganz klar.

12./X. seit dem 10. Abend nicht mehr eingespritzt, Bier getrunken; kein Secret, keine Fäden im Urin, der klar erscheint; beim Stehenlassen bildet sich ein leichtes Sediment, das centrifugirt noch eine geringe Anzahl G. C. in und ausser Zellen aufweist. Irrigation mit Arg. nitr. 1:750.

17./X. seit dem 13. nicht eingespritzt, wieder Bier getrunken, eine mit Mühe erhaltene Spur von Secret zeigt nur Epithelien, keinerlei Coccen, Urin völlig klar, in der 1. Portion eine feine Faser. Inj. Albargrtn $2 \%$ in vordere Urethra.

26./X. hat noch einmal eingespritzt; kein Secret. Urin klar mit ein Paar kleinen Fasern, die grösstentheils aus Epithelien besteben, nur spärliche Eiterzellen, keine Coccen.

27./XII. Patient bemerkt heute c. 12 Tage nach Coitus etwas schleimiges Secret, dass einige Eiterzellen aber keine Coccen zeigt, Urin klar, in der 1. Portion einige ganz feine Fäden. Inject. 10\% Airol, darauf nichts mebr gesehen.

Trotzdem, dass hier schon 6 Tage Symptome der Infection vorhanden gewesen waren und zeitweilig eine Urethritis posterior vorhanden gewesen war, verschwanden innerhalb 18 Tagen nach Beginn der Behandlung Secretion und Coccen vollständig und zwar bei nur sehr wenig veränderter Lebensweise des Patienten, einschliesslich mässigen Genusses geistiger Getränke.

Abgesehen von dem beinahe vollständigen Fehlen subjectiver Beschwerden können die Resultate in diesen beiden Fällen als nicht unbefriedigende hingestellt werden, da die Dauer der Krankheit doch noch beträchtlich hinter den üblichen 4 bis 6 Wochen zurückbleibt.

Weit günstiger jedoch gestalten sich die Verhältnisse und weit zahlreicher sind die Erfolge in Fällen von frischer Infection bei Patienten, die bereits früher mehr wenige lange dauernde Gonorrhoen durchgemacht hatten, zum grossen Theile 
solchen, welche wegen derselben in meiner Behandlung gestanden hatten. Solche Patienten durch die Erfahrung belehrt, dass die Gonorrhoe doch eine recht unangenehme Krankheit werden könne, beobachten die ersten Zeichen derselben genau, wissen dieselben auch sogleich richtig zu deuten und sind durch dieselben genügend beunruhigt, um sofort den Arzt aufusuchen. Wie schon erwähnt zeigen diese Fälle allerdings an und für sich eine gewisse Neigung zu einem milderen Verlauf, selbst wenn Jahre seit der letzten Gonorrhoeerkrankung verflossen waren, keineswegs aber die Neigung spontan zu verschwinden, vielmehr ist die Tendenz, die hintere Harnröhre zu befallen und in chronische Formen überzugehen, eine recht bedeutende. Die Incubationszeit ist nach meinen Beobachtungen in der Regel eine kürzere und habe ich die Erscheinungen von Entzündung, insbesondere das Auftreten von Gonococcen schon 24 bis 36 Stunden nach dem inficirenden Coitus feststellen können. So ist es mir möglich geworden, eine ziemliche Anzahl von Patienten innerhalb 3 bis 4 Tagen nach erfolgter Infection in Behandlung zu bekommen, zu einer Zeit, wo die localen Entzündungserscheinungen wie Röthe und Schwellung der Schleimhaut des Orificium, Brennen beim Uriniren, Jucken in der vordern Harnröhre nur sehr gering oder gar nicht vorhanden waren. Unter solchen Umständen schlage ich dann gewöhnlich folgendes Verfahren ein: Nach Entleerung der Blase wird sofort eine Einspritzung von einer Silberlösung vorgenommen, wie Argent. nitr. $1 / 4$ bis $1 / 2 \%$, Protargol $2-4 \%$ oder Albargin $2 \%$ in den allerletzten Wochen auch $5 \%$ und zwar wird die Flüssigkeit etwa 3 bis 4 Zoll ( 8 bis 10 Centimeter) hinter der Harnröhrenmündung, also nicht einmal ganz im Bulbus entleert. Meist wird zuerst die schwächere Lösung probirt, und wenn dieselbe nur unbedeutendes Brennen verursacht, wird eine zweite Spritze mit der stärkeren Lösung zugefügt; die Flüssigkeit wird einige Minuten in der Harnröhre zurückgehalten und durch vorsichtiges Zusammendrücken des Penis von aussen her möglichst in der ganzen vordern Harnröhrenpartie zur Vertheilung gebracht. Erscheint der Patient in der Vormittagssprechstunde, so macht er dann vor dem Abendessen ( 6 bis 7 Uhr) und vor dem Schlafengehen eine Einspritzung mit der 
B. P. Z. Lösung, aber nicht am andern Morgen, sondern stellt sich wieder in der Sprechstunde ein, um wiederum eine Silbereinspritzung meist mit der stärkeren der oben genannten Lösungen applicirt zu bekommen. Am Abend verfährt er wie des Tages zuvor, desgleichen am 3 . Tage nach erneuerter Silberinjection. Diese wird am 4. Tage ausgesetzt, dafür $3 \mathrm{mal}$ die eigne Einspritzung gebraucht. Am 5. Morgen wieder Silberinjection, Fortsetzung der andern Finspritzung bis zum 7. Tage Morgens, von da ab keinerlei Behandlung bis zum 8. Tage Früh, wann die Untersuchung womöglich vor dem Entleeren des Nachturins vorzunehmen. Auch wenn keine Erscheinungen von Gonorrhoe mehr vorhanden sind, wird die Silberinjection nochmals wiederholt, darauf aber alle Behandlung ausgesetzt, es müssten denn neverdings Ausfluss oder Gonococcen auftreten. Recbt häufig aber ist schon vom 3. Tage ab kein Secret mehr vorhanden oder wenn noch in geringer Menge ausdrückbar, frei von Gonococcen. Trotzdem halte ich die Verlängerung der Behandlung in jedem Falle für rathsam, obwohl einige Beobachtungen, in denen die Patienten sich selbst vom 2. Tage ab als geheilt betrachteten und die Behandlung abbrachen, den Beweis lieferten, dass in der That die Gonococcen und mit ibnen die Absonderung definitiv beseitigt waren und blieben. Unter einer Anzahl von solchen Fällen, die ich kürzlich zusammenstellte behufs einer Vergleichung der Wirksamkeit des Albargins, waren 15, die innerhalb 24 bis 36 Stunden nach der Beobachtung der ersten Symptome zur Behandlung kamen. Von diesen wurden 11 innerhalb von 8 Tagen als geheilt entlassen und blieben ohne jede weitere Behandlung jedenfalls wochenlang frei von irgend welchen Symptomen. Die Durchschnittszahl der (Doppel-)Injectionen des Silbersalzes $(2 \%)$ bei diesen 11 Patienten betrug nur 4. Ein Patient erhielt nur 2, zwei nur 3, fünf erhielten je 4 und drei je 5. Die Einspritzungen vertheilen sich so, dass nach dem ersten Tage 10 auf den 2. Tag, 10 auf den 3., eine auf den 4., sechs auf den 5., zwei auf den 6. und 3 auf den 8. Tag der Behandlung kamen. In den übrigen 4 Fällen gelang es nicht die Krankheit in so kurzer Zeit zum Verschwinden zu bringen, obwohl acute Erscheinungen entweder gar nicht auftraten oder rasch beseitigt wurden. Nach längerer 
erfolgloser Behandlung wurde in diesen Fällen die endoskopische Untersuchung vorgenommen und in sämmtlichen gelang es als UTsache des anhaltenden Ausflusses und Gonococcenbefundes umschriebene locale Veränderungen der Schleimhaut, wahrscheinlich Folgezustände früherer Erkrankungen nachzuweisen, die den Gonococcen besonders günstige Ansiedlungsstellen und Schlupfwinkel geboten zu haben schienen. Einer dieser Patienten, der bei seiner letzten Gonorrhoe längere Zeit an Rheurnatismus gelitten hatte, blieb von dieser Complication völlig verschont, bei keinem der Kranken kam es zu Urethritis posterior, Epididymitis oder andern Complicationen. $\mathrm{Da}$ der Zustand der Schleimhaut meist schon nach kurzer Behandlung eine endoskopische Untersuchung vorzunehmen gestattet ohne nachtheilige Folgen, pflege ich dieselbe nicht zu lange aufzuschieben, sondern längstens nach 2 bis 3 Wochen die Ursache der verzögerten Heilung direct aufzusuchen.

Kommt der Kranke in Behandlung, wenn die Symptome der Gonorrhoe schon länger als 3,4 oder höchstens 5 Tage bestehen, so sehe ich von jedem Versuche, die Krankheit rasch zu beseitigen, von vornherein $a b$ und begnüge mich damit, die Entzündungserscheinungen auf einem mässigen Grade zu erhalten, resp. auf einen solchen herabzusetzen. Zu diesem Zwecke werden Silbereinspritzungen nur jeden 3. oder 4. Tag vorgenommen, dazwischen aber adstringirende Einspritzungen seitens des Patienten vorgenommen, zuweilen auch innere Mittel wie 0l. Santali zu Hilfe genommen. Hier kommen nun im Allgemeinen schwächere Silberlösungen zur Verwendung, gelegentlich auch Thallinum sulphuricum und Ichthyol, doch ist es manchmal überraschend, wie gelegentlich auch bei reichlichem eitrigen Ausfluss 1-2\% Protargol, 1\% Albargin, $1 / \mathrm{s} \%$ Argent. nitric. nicht nur gut vertragen werden, sondern auch sofortige Abnahme der Secretion bewirken, oft auch ohne gleichzeitigen Gebrauch der Adstringentien. Meist beginnt dann nach 3 bis 4 Tagen der Ausfluss wieder zuzunehmen, dann wird die Silberlösung schon in stärkerem Procentgehalt wieder injicirt und so allmälig eine Abnahme der Symptome und eine Verlängerung der Pausen zwischen den stärkeren Applicationen erzielt.

Bei sebr heftigen Entzündungsgraden ist jedenfalls grosse 
Die Bohaudlung der acuten und subacuten Gonorrhoea etc. 419

Vorsicht nöthig und bei den ganz virulenten Fällen, die mir allerdings jetzt in der Privatpraxis recht selten zu Gesicht kommen, enthalte ich mich auch zunächst aller örtlicher Behandlung. Unter solchen Umständen habe ich seit Jahren mit vorzüglichem Erfolg innerlich das Natr. salicylic. mit 'oder ohne Zusatz von Extract. Belladonnae angewandt, in mässigen Dosen aber reichlich verdünnt in Wasser oder alkalischen Mineralwässern genonmen, etwa nach folgender Verordnung:

Natr. salicylic. $12 \cdot 0-15 \cdot 0$

(Extr. Belladonnae 0.15-0.20)

Aquae Menthae piperitae 100.0 .

SDS. 2-3stündlich einen Theelöffel voll in Wasser zu nehmen (also etwa 50 bis 60 Centigrammes pro dosi). Binnen weniger Tage pflegt eine bedeutende Herabsetzung der Empfindlichkeit und der Anschwellung der Schleimhaut beziehentlich des ganzen Penis einzutreten, auch das Secret wird dünner und weniger gelb gefärbt; die vermehrte Diurese ist wohl mehr dem reichlichen Wassergenuss zuzuschreiben. Diese Medication vereinigt die antiseptische Wirkung der Salicylsäure mit der gewöhnlich in solchen Zuständen angestrebten Verminderung des Säuregrades des Urins und wirkt kaum schlechter auf den Magen als die üblichen Verordnungen kohlensaurer oder citronensaurer Kalisalze oder des Leinsamenthees. In solchen Fällen ist natürlich die Ruhestellung des Patienten selbst wie des erkrankten Gliedes von grosser Wichtigkeit, während ich in den meisten anderen Fällen die Patienten unter gewissen Einschränkungen ruhig ihren Geschäften nachgehen lasse.

Einige Beispiele, ohne besondere Wahl meinen Krankenjournalen entnommen, mögen in Kürze den Verlauf unter der vorgeschlagenen Behandlung veranschaulichen.

Fall 4. M. S., gegenwärtig 65 Jahre alt, war bereits 1882 wegen hartnäckiger Gonorrhoe in meiner Behandlung, 1886 mit Syphilis inficirt, 17. September1888 kam wegen Gonorrhoe in Behandlung, die mit Irrigationen von Kali hypermanganicum und Sublimat behandelt, am 18. October geheilt war.

17. Juni 1895. Gonorrhoe, behandelt mit Inj. von Arg. $1 / 2$ bis $1 \%$; am 28. noch einige freie Diplococen, am 1. Juli Symptome von Urethritis posterior acuta durch tiefe Instillationen von Arg. nitr., am 6. Juli anscheinend dauernd beseitigt. Am 29. August vorübergehend Secret ohne Coccen, Recidiv(?), am 14. September wieder Behandlung mit Arg. 
nitr. $1 / 4$ bis $1 / 2 \%$. Am 4 . October waren seit einigen Tagen alle Symptome verschwunden gewesen.

4. October 1899. Seit heute Morgen Ausfluss bemerkt mit reichlichen Gonococcen, Inj. Protargol $2 \%$. Bis zum 15. October wurden 5mal Injectionen mit Protargol $2 \%$ wiederholt, Secret war überhaupt nicht wieder beobachtet worden.

11. August 1900. Hat seit gestern Ausfluss bemerkt, 4 Tage nach Coitus, reichliche Gonococcen. Inj. Protargol 2 und $4 \%$, desgl. am 12., 13. und 14. Kein Secret mehr beobachtet.

30. Juni 1901. Während Abwesenheit im Westen seit 12 Tagen Gonorrhoe mit gewöhnlichen Einspritzungen and Ol. Santal. behandelt. Injectionen von Albargin $2 \%$ bewirkten rasche Abnahme aber kein Verschwinden der Gonococcen oder der Symptome. Endoskopische Untersuchung am 16. August zeigte noch ziemlich acute Entzündungszustände in der vordern pendula mit beginnender Stricturbildung, die eine längere locale Behandlung bis zur völligen Beseitigung nöthig machten.

Fall V. C. R. 36 Jahre alt, hat verschiedene, zum Theil hartnäckige Gonorrhoen durchgemacht, zuletzt Anfang 1898.

4. Februar 1899, bemerkt seit 2 Tagen Ausfluss, Gonococcen nicht nachweisbar, dennoch Inj. Protargol $2 \%$. Am 7. Februar typische Gonococcen, Inj. Protargol 2 und $4 \%$. Am 13. Februar nichts mehr zu sehen. Am 16. Februar Rückfall, nach Injection von Protargol am 16., 17., 19., 21., 24., am 27. noch einige Coccen, 5. März seit einigen Tagen kein Secret mehr beobachtet.

21. Juli 1901. Seit 3 Tagen Absonderung, wegen Abwesenheit auf dem Lande erst heute erschienen, typische Gc. Albargin $2 \%$; am 23 . und 24. kein Secret, am 25. Spur mit einigen Gc., desgleichen am 26. und 29. Am 1. August noch einige Diplococcen ausser Zellen. 5. August: Seit 2 Tagen nicht mehr eingespritzt, keine Gc., am 10. August ohne Behandlung seit dem 5. eine Spur schleimigen Secrets. Epithelien, keine Coccen.

Fall VI. E. S. 39 Jahre alt, Anfangs 1900 chron. Gon., bis April geheilt, 1901 im Februar bemerkte der sehr ängstliche Patient etwas Absonderung, keine Coccen, durch eine Injection von Airol 10\% beseitigt.

16. Juli 1901. Gestern Abend, 3 Tage nach Coitus Secret bemerkt, jetzt typische Gonococcen, Albargin $2 \% ; 17 . /$ VII.: Spur von Schleim, frei von Gc., am 18./VII. ein wenig Secret mit ein Paar zweifelhaften Diplococcen, 20./VII. Spur von Schleim, grösstentheils Epithelien, keine Gonococcen.

Fall VII. W. L., 24. Jahre alt, besuchte mich am 22. August 1901, weil er im Juli vor seiner Abreise von Dentschland an Gonorrhoe behandelt worden war, um sich auf Rath seines früheren Arztes zu vergewissern, ob die Heilung eine vollständige gewesen sei. Untersuchung am Morgen vor der Entleerung des Nachturins ergab vollständig negative, Befund. 
21. September. Pat. bemerkt seit 3 Tagen, eine Woche nach Coitus, Ausfluss mit typischen Gonococcen. Inj. Protargol $2 \%$, desgleichen am 22. und 23, bei sehr geringem Secret; 24./IX. etwas wässeriges Secret obne Gc. Inj. Arg. nitr. $1 / 4$ u. $1 / 2 \%$. Am 26./IX. etwas klares Secret ohne Gc., Inj. Arg. nitr. $1 \frac{2}{2} \%$. 2. October: seit 3 Tagen keine Spur von Absonderung.

Fall VIII. J. W. 29 Jahre alt, letzte Gonorrhoe vor 10 Jahren, letzter Coitus am 21. December 1900, seit dem 23. Absonderung. 25. December: typische Gc. Inj. Protargol $2 \%$. 26. December: trotz Pollation wenig Secret, am 27. nur Spuren, desgl. am 29. Inj. Arg. nitr. $1 / 4$ u. $1 / 2 \%$. 31. December kein Secret. 3. Jänner 1901.: Pat. will trotz Trinkens und Tanzens nichts mehr von Ausfluss bemerkt haben, doch findet sich ein gelbliches Tröpfchen, welches noch einige Gc.-haltige Zellen aufweist. Inj. Arg. nitr. $1 / 2 \%$.

7./I. 1901. Ausfluss war verschwunden, nach Trinken von Whiskey heute wieder etwas eitriges Secret mit typischen Gc. Inj. Protargol $4 \%$.

9./1. Spur grauen Secrets. Inj. Protargol $4 \%$.

12./l. Trotz aller möglichen Provocation kein Secret, im klaren Urin ein Paar feine Fäden, Inj. Protargol $4 \%$, von Morgen ab keine Behandlung.

16./I. Pat. hat seither nichts mehr gesehen.

F all IX. D. K. 23 Jahre alt, vor 2 Jahren zum ersten Male Gonorrhoe, bemerkt 29. April 1901 nach Coitus am 27. Ausfluss, zähes schleimiges Secret mit typischen Ge. Iaj. Albargin $2 \%$ ziemlich brennend. Inj. B. P. Z.

1. Mai. 2 Stunden nach Uriniren kein Secret, geringe Trübung der ersten Urinportion, Albargin $2 \%$ kaum empfindlich.

6. Mai. Pat. hat bis gestern Morgen eingespritzt, nach 10stündigem Anhalten des Urins kein Secret, Urin ganz klar. Inj. Albargin $2 \%$, keine weitere Behandlung.

Fall X. J. H. 29 Jahre alt. 1898 längere Zeit an chronischer Gonorrhoe behandelt und geheilt. 8. Mai 1901 nach starkem Trinken gestern Morgen Coitus, gestern Abend schon verdächtige Symptome, heute deutliches Secrat mit typischen Ge. Inj. Albargin $2 \%$, selbst keine Einspritzung zu machen. 9. Mai, am Morgen Spur von Secret, aber keine Gc. Inj. Albargin $2 \%$ verursacht etwas Bremen.

10./V. am Morgen ganz geringe Menge Secret, Eiterzellen aber keine Gc. Albargin $2 \%$.

12./V. Befund der gleiche. Albargin $2 \%$, nicht empfindlich.

Am 4. Juli erschien Pat. wieder mit ziemlich reichliche Gc. enthaltender Absonderung, die er gestern nach Coitus am 30. Juni bemerkt batte. Er war vorher 3 Wochen auf Reisen gewesen und hatte trotz wiederholtem Coitus keine Absonderung bemerkt. Albargin $2 \%$, desgl. am 5./VII. und 6./VII, nur Spuren von Secret.

9. Juli, am Morgen vor Entleerung des Nachturins orificium verklebt, dahinter ein grauer Schleimtropfen, der noch Eiterzellen aber keine Gre. oder andere Bakterien aufweist. Inj. Albargin $2 \%$

13. Juli. Trotz Trinkens am Morgen nur Spur von Schleim ohne Gc. aber noch einige Eiterzellen, im Urin eine lockere Schleimfaser. Albargin $2 \%$ absolut unempfindlich. Keine weitere Behandlung.

Derartige Beobachtungen könnte ich noch in ziemlicher Menge 
anfübren, dieselben sind keine Ausnahmen, sondern repräsentiren den üblichen Verlauf, auf den ich roit ziemlicher Sicherheit rechnen kann, wenn die Behandlung in den ersten Tagen genan durchgeführt wird.

Bei den von mir als subacute bezeicbneten Fällen, in denen der Ausfluss scheinbar verschwindet, aber etwas Secret sich aus dem hintern Theil der pars pendula ausdrücken lässt, oder wo der Ausfluss nach dem Aussetzen der Behandlung rasch wieder sich einstellt, werden, wie bereits eingangs beschrieben, die Silbereinspritzungen anfangs jeden 3. bis 4. Tag gemacht, während der Patient eine der adstringirenden Einspritzungen 3 bis 2 mal täglich selbst macht, aber 24 Stuvden vor dem Besuch der Sprechstunde damit aussetzt. Nach und aach werden die Pausen zwischen den Silberinjectionen bis zu 7 bis 8 Tagen verlängert, während die adstringirenden Einspritzungen des Patienten gleichzeitig auf 2 und 1 pro die verringert und zuletzt ganz weggelassen werden. Die folgenden 2 Fälle mögen genügen, um die Erfolge der Behandlung zu veranschaulichen.

F all XI. A. J. G., 27 Jahre, verheiratet, vorher nie geschlechtlich krank gewesen, bemerkte am 3. Juli 1900 Ausfluss und begab sich sofort in ärztliche Behandlung. Dieselbe bestand anfangs in innerlicher Verab. reichung von 01. Sant., später in Einspritzungen. Die Secretion hatte verschiedene Male ganz aufgebört, war aber immer wieder gekommen. Das Secret soll anfangs sehr reichlich aber frei von Gonococcen gewesen sein. Inzwischen hatte Patient auf Anrathen seines Arztes, der iln für geheilt erklärt hatte, mit seiner Frau cohabitirt und dieselbe angesteckt, später war auch seine כjährige Tochter inficirt worden. Als Patient mich zum ersten Male consultirte, hatte er kurz vorher die Blase entleert, auch eine Sonde eingefübrt, so dass ich weder Secret noch Urin erhalten konnte und die Untersuchung auf den nächsten Tag verschob. Am 23. September fand ich nun nnerwartet reichlichen Ausfluss mit zallreichen Gc.; die erste Urinportion trüb, die zweite klar, kein Brennen beim Uriniren. Inj. Protargol 2\% in Bulbus, 2 Spritzen, wenig Brennen. Inj. B. P. Z.

27. September. Ausfluss hat sofort aufgehört, kein Secret, Urin durchaus klar mit einigen dünnen Fäden, Inj. Arg. nitr. $1 / 4$ und $1 / 2 \%$ in Bulbus, mässig empfindlich, Einspritzung B. P. Z. und jeden zweiten Abend 1\% Protargol.

2. October. Geht gut, keine Spur von Secret, absolut klarer Urin, keine Fäden. Inj. Arg. $1 / 4$ und $1 / 2 \%$, noch etwas empfindlich, Einspritzungen wie vorher fortzusetzen.

11. Oct. Trotzdem dass Pat. am 3. Fieber und Glicderschmerzen ohne nachweisbare locale Ursache bekam und sich mit Tiuct. Warburg und türkischem Bad behandelt hatte, kein Secret, Urin völlig klar, kein Eiweiss. Inj. Arg. nitr. $1 / 4$ und $1 / 2 \%$, soll nach 2 Tagen seine Einspritzungen aufgeben, eventuell Bier trinken.

16. Oct. Pat. kommt mit allerlei Klagen, doch lässt sich im urogenitalen Tractus kejne Störung nachweisen. Inj. Arg. nitric. $1 / 4$ u. $1 / 2 \%$ noch 2 Tage einzuspritzen. Da er als Kind Diabetes gehabt haben soll, wird Urin mit negativem Erfolg durch Gährungsprobe auf Zucker untersucht.

19. Oct. Klagt wieder über Schmerzen in Gliedern und Fieber, zeigt aber normale Temperatur und Puls, keine Erscheinungen an der Harnröhre, daher keine locale Behandlung, Phenacetin 0.3 3mal täglich. 25 Oct. Hat jetat seit einer Woche alle Bebandlung ausgesetzt, in der Harnröhre eine geringe Menge farbloser, wässriger Flüssigkeit, anscheinend nur Urin; der entleerte Urin völlig klar und frei von Fäden, spec. Gewicht 1005, keine Reaction mit Fehling; Coitus mit Frau war ohne irgend welche nachtheilige Folgen.

Der Kranke war augenscheinlich schon nach der 3. Injection am 
2. October geheilt gewesen, doch wurde der Sicherbeit wegen die Behandlung fortgesetzt.

F all XiI. R. W. E., 22 Jahre alt, Student, leidet seit 3 Monaten an seiner 3. Gon. keine der früheren Attaquen war besonders schwer oder durch Complicationen ausgezeichnet. Der Ausfluss schien mehrfach beseitigt, kehrte aber immer 2 Tage nach Aussetzen der Behandlung zurück; hat Einspritzungen und innere Mittel, zuletzt Methylenb]au gebraucht.

23./IV. Reichliches eitriges Secret mit zahlreichen mit Gc. strotzend gefüllten Eiterzellen, 2. Urin klar, enger Meatus. Inj. Albargin 1\% u. $2 \%$ in Bulbus, mässig empfindlich, an einer Stelle in der Mitte der pendula etwas Widerstand. Einspritzungen B. P. Z.

26./IY. Seit gestern Morgen kein Secret mehr beobachtet, jetzt orific. ganz trocken, Urin noch ein wenig trüb, Inj. Albarg. $2 \%$ bis in membranacea, Widerstand in der Pendula nicht zu bemerken.

29./IV. Seit dem 27. nicht eingespritzt, kein Secret, ein wenig grauer Schleim zeigt Epithelien, keine Gc. Urin noch wenig trüb. Inj. Albarg. $2 \%$. 2 Spritzen in Bulbus, nach heute Abend keine Einspritzung mehr. Urotropin $0.53 \mathrm{mal}$ pro die.

2./V. Seit dem 30. nur Urotropin, hat Rudern angefangen, in der Nacht vorher Pollution, eine Spur Schleim zeigt einige Eiterzellen neben Plattenepithelien, keine Gc., Úrin 1. Portion noch etwas trüb, 2. völlig klar, eine Inj. Albarg. 2\%. Einspritzung noch beute Abend. Urotropin noch einige Tage einzunehmen.

Zum Schlusse möchte ich besonders betonen, dass ich die im Vorstehenden dargestellte Behandlungsmethode keineswegs als eine abortive empfohlen haben möchte, obwohl sie in der That in einer Anzahl von Fällen in überaus kurzer Zeit die Infection mit Gonococcen dauernd $\mathrm{zu}$ beseitigen vermocht hat. Was ich für dieselbe in Anspruch nehme ist, dass sie im Stande ist in einer grossen Anzahl von Fällen, namentlich wenn sie frühzeitig in Behandlung kommen, die Gonorrhoe in kürzerer Zeit als andere Methoden zu heilen oder wenigstens die Symptome derselben auf ein sehr geringes Mass zu reduciren, die mit derselben verbundenen Unannehmlichkeiten zum grossen Theil zu vermeiden, die Weiterverbreitung auf die urethra posterior sowie Complicationen seitens der Blase, Prostata und Nebenhoden einzuschränken; dass sie ausserordentlich einfach, namentlich viel weniger umständlich als die Janet'sche Spülmethode ist. Dass bei derselben die Hauptsache der Behandlung in die Hände des Arztes selbst gelegt ist, scheint mir auch im Interesse des Patienten eher ein Vortheil als ein Nachtheil zu sein, da sie ersterem eine gute Controle über den Kranken und die Krankheit gewährt. Wenn dadurch dem Pat. vielleicht im Anfang etwas grössere pecuniäre Opfer zugemuthet werden, so wird dies wohl reichlich durch die Abkürzung der 
Behandlung aufgewogen. Wer mit der sogenannten zuwartenden Methode zufrieden ist, wird sich schwerlich für das etwas eingreifendere Vorgehen begeistern. :Ich muss gestehen, dass es mir früher immer ein demüthigendes und bedrückendes Gefühl verursacht hat, die Patienten wochenlang mit innern Mitteln oder mit Einspritzungen, an deren Wirksamkeit wir selbst wenig glauben, zu behandeln und kann ich es nicht so unbegreiflich finden, wenn viele Kranke sich selbst die bekannten Trippermedicinen rerschaffen, die Einspritzung irgend eines guten Freundes auf eigene Faust benutzen oder sich in die Hände von Pfuschern begeben und die Aerzte erst aufsuchen, wenn die Krankheit die hergebrachte Dauer zu überschreiten beginnt oder Complicationen eintreten. Ich stimme vollständig überein mit Scholz (Ueber die Bekämpfung der Geschlechtskrankheiten unter den Studenten, Münchener Med. Woch. 1901, Nr. 5), dass die Kranken sich bald gewöhnen, bei Zeiten ärztliche Hilfe aufzusuchen, wenn sie wissen, dass ernste und nicht erfolglose Anstrengungen gemacht werden, die Krankheit zu bekämpfen und abzukürzen, selbst wenn nicht immer so schnell oder augenblicklich der Erfolg eintritt. Ich kann versichern, dass Patienten, die einmal die Erfahrung gemacht haben, dass sie, ohne eigentlich viel ron der Krankheit merken, in 8 bis 10 Tagen oder gar in noch kürzerer Zeit von derselben befreit wurden, keine Zeit verlieren werden, bei dem ersten leisesten Anzeichen, die ärztliche Hilfe aufzusuchen, oder wo sie Gelegenheit haben, ihre Bekannten und Freunde veranlassen das Gleiche zu thun. Indem ich die Behandlungsmethode den Collegen zu vorurtheilsfreier Prüfung empfehle, vor Allem allerdings solchen, die sich schon einige Erfahrung in der Beurtheilung und Behandlung der Gonorrhoe angeeignet haben, bin ich mir wohl bewusst, dass sie sich nicht in den Händen Aller wirksam erweisen mag, doch bin ich überzeugt, dass sie sich manche Freunde erwerben und neben andern Methoden eine gewisse Verbreitung gewinnen wird. 